\title{
L'incoerenza creativa nella narrativa francese contemporanea, M. Majorano (dir.)
}

\section{Francesca Dainese}

\section{(2) OpenEdition}

1 Journals

\section{Edizione digitale}

URL: http://journals.openedition.org/studifrancesi/10605

DOI: 10.4000/studifrancesi. 10605

ISSN: 2421-5856

\section{Editore}

Rosenberg \& Sellier

\section{Edizione cartacea}

Data di pubblicazione: 1 décembre 2017

Paginazione: 587-588

ISSN: 0039-2944

\section{Notizia bibliografica digitale}

Francesca Dainese, «L'incoerenza creativa nella narrativa francese contemporanea, M. Majorano (dir.)»,

Studi Francesi [Online], 183 (LXI | III) | 2017, online dal 01 février 2018, consultato il 21 janvier 2021.

URL: http://journals.openedition.org/studifrancesi/10605; DOI: https://doi.org/10.4000/studifrancesi. 10605

Questo documento è stato generato automaticamente il 21 janvier 2021.

\section{(c)}

Studi Francesi è distribuita con Licenza Creative Commons Attribuzione - Non commerciale - Non opere derivate 4.0 Internazionale. 


\title{
L'incoerenza creativa nella narrativa francese contemporanea, M. Majorano (dir.)
}

\author{
Francesca Dainese
}

\section{NOTIZIA}

L'incoerenza creativa nella narrativa francese contemporanea, a cura di Matteo MAJORANO, Macerata, Quodlibet Studio, 2016, «Lettere. Ultracontemporanea», 202 pp.

1 L'incoerenza? «Un talismano contro la sorte» e una «risorsa per il romanzo». Questa la chiave di lettura che apre la miscellanea sul «disordine», stilistico e strutturale, che sembra aver invaso la narrativa francese contemporanea. «Entrare in un romanzo è come entrare in un edificio ignoto", scrive M. MAJORANo nel saggio introduttivo (L'incoerenza e la letteratura, pp. 9-25). Con Perec, lo studioso raccomanda "precauzione» e «spirito di osservazione» di fronte alle «sgrammaticature» dell'autore, padrone di casa poco cerimonioso, che, pagina dopo pagina, offre all'ospite-lettore tutti i tranelli del suo «libero arbitrio».

Davanti all'impossibilità, già denunciata da Platone, di proferire «ragionamenti sempre [...] "concordi ed esatti"», Elio FRANZINI articola il suo discorso sulla verità, letteraria ed artistica in generale, interrogandosi sul concetto di «errore coerente», una «premeditat[a] trasgression[e] di un codice naturalistico-imitativo del mondo, a fini espressivi» (L'incoerenza creativa: un problema moderno?, pp. 27-37). Ma l'incoerenza non riguarda soltanto sintassi e semantica per Akira MIZUBAYASHI. Nel suo intervento Pourquoi écrire dans une langue qui n'est pas la mienne? (pp. 39-48) egli identifica il primo tratto di incoerenza del suo essere scrittore nel «vouloir faire de la littérature dans une langue doublement éloignée, nativement d'une part et géographiquement et culturellement de l'autre». 
3 Michael SCHNEIDER, in Le Roman est-il quantique? (pp. 49-56), si propone invece questo campo di riflessione: «si le roman, comme l'amour lui-même, n'était pas affaire de mémoire et de temps, mais d'une co-présence d'éléments intriqués? D'une unité de matière en quelque sorte hors du temps et de l'espace?». A suo parere, il romanzo e in particolare il romanzo d'amore, «comme la physique quantique, met en cause la réalité de la réalité».

4 L'opera della scrittrice Gaëlle Josse è fatta di accordi dissonanti che compongono la sua «musique personelle». A proposito dei suoi romanzi, uno su tutti Nos vies désaccordées, l'autrice parla delle incoerenze che attraversano il suo vissuto (e quello dei suoi personaggi) come delle «ruptures». La vera libertà creatrice, afferma, inizia nel momento in cui l'autore smette di voler dominare a tutti costi la propria opera (L'incohérence narrative, ou les étonnantes tribulations d'un inconscient au travail, pp. 57-66).

5 Marie Thérèse JACQUET, in L'incohérence: du superflu à l'incontournable (pp. 67-81), riflette sul processo di omeostasi che il lettore mette in atto di fronte alle incoerenze (spesso sapientemente pianificate) del suo autore. Tra empatia e decifrazione dell'enigma, la studiosa fa riferimento, tra le altre, all'opera di Modiano, Echenoz, Egloff, Oster e Houellebecq. Di quest'ultimo parla anche Matteo MAJORANO ne L'incohérence créatrice: de Beckett à Houellebecq (pp. 83-98), saggio in cui da Mercier et Camier a Soumission, egli identifica il ruolo dell'incoerenza come «l'un des noyaux dynamiques centraux, un des moteurs qui contribuent à organiser un roman», a livello tanto tematico quanto stilistico e formale.

6 L'incoerenza, secondo Dominique VIART (Incohérences narratives du fait divers, pp. 99-113), sta alla letteratura come un fattore «di scandalo» e allo stesso tempo «di evidenza». Ma che succede quando è la materia stessa a far esplodere la forma romanzesca e non necessariamente il suo autore? E quando «le narrateur s'interroge en effet en tant que narrateur, dans l'exercice de sa narration?». Viart afferma, con Bourdieu, che «les faits divers font diversion»: non ci resta che accettare, quindi, riga dopo riga, «les effets d'inchoérence apparente» di cui è disseminato il reale e di cuil l'autore è vittima, quanto il suo lettore.

7 La percezione dell'incoerenza nella letteratura contemporanea viene analizzata da Alexandre GEFEN come la reazione a un modello di coerenza prestabilito: in questo senso, essa rientra nella più ampia categoria del postmoderno. Tuttavia, lo studioso mostra come, con il ritorno al reale degli scrittori della generazione degli Incultes e dei néo-réalistes, l'eterogeneità sia la nuova protagonista della letteratura, sullo sfondo delle teorie filosofiche del «nouveau réalisme» e del «réalisme spéculatif» (Le monde n'existe pas: "le nouveau réalisme" de la littérature française contemporaine, pp. 115-125).

8 Dalla «coerenza letteraria» all'«incoerenza sociale»: Giusi Alessandra FALCO analizza l'opera di Annie Ernaux alla luce di un movimento di rottura, di trasgressione, rispetto al momento storico cui i suoi romanzi si riferiscono, ovvero gli anni Sessanta-Settanta. La studiosa, a partire dai racconti La femme gelée e Une femme, propone il concetto di «incohérence de genre» (Annie Ernaux: la cohérence littéraire de l'incohérence féminine, pp. 127-139).

9 Per Marinella TERMITE (Caprices de la narration. Les contrepoids d'Emmanuel Carrère, pp.141-152) l'incoerenza si fa "pratique scripturaire, loin de tout reflux de l'inconscient», di fronte al «corto-circuito» del reale. L'«hétérogénéité, la fragmentation, la saturation» sono, secondo la studiosa, «meccanismi di incoerenza» 
che Carrère mette in scena in tutti i suoi romanzi, da L'amie du jaguar a Le Royaume. Sjef HOUPPERMANS riflette invece sull'incoerenza narrativa che scaturisce dall'incoerenza dell'inconscio delle psicotiche protagoniste di Julia Deck in Vivianne Elisabeth Fauville e Le triangle d'hiver (Julia Deck, incohérence psychique et logique textuelle, pp.153-165). In Tristesse de la terre, Éric Vuillard ci trascina infine nell'immaginario del selvaggio West e delle ultime guerre indiane. Siamo di fronte a un palcoscenico d'eccezione, spettacolare, di cui il regista è Buffalo Bill Cody. Pierre sCHOENTJES analizza questo romanzo sotto molteplici prospettive, mettendo in rilievo soprattutto l'importanza del capitolo finale: «une rupture qui frise l'incohérence» e che cambia tutta la prospettiva della narrazione («Bill l'imposteur»? Éric Vuillard et les incongruités révélatrices de “Tristesse de la terre", pp. 167-182).

10 Secondo Christian viGUié, ne L'incohérence créative: une possibilité artistique ou l'histoire d'un malentendu entre l'œuvre et le monde? (pp. 183-190), «l'écrivain serait une sorte de voyant aux yeux crevés» di fronte al problema dell'incoerenza creativa nella prosa letteraria francese. La coerenza, dice, è un'invenzione, un punto di arrivo, più che un processo: «Je considère la cohérence comme une invention, comme un procédé. Elle tient dans sa main un jeu des clés que l'écrivain devra dérober». E l'incoerenza dunque? C'est «l'écho de l'errance».

11 In conclusione, per quanto sembri impossibile tracciare un ritratto coerente dell'incoerenza, questa raccolta ha il merito di analizzare le sue risultanze letterarie nel panorama francese contemporaneo sotto molteplici (e appassionanti) punti di vista. 